\title{
Plasma tocopherols and carotenes are decreased in Spanish metabolically unhealthy children and adolescents independently of obesity
}

\author{
Azahara I. Rupérez ${ }^{1}$, Gloria Bueno ${ }^{2,3}$, Rosaura Leis ${ }^{4,3}$, Mercedes Gil-Campos ${ }^{5,3}$ Ángel Gil ${ }^{6,3}$, \\ Luis A. Moreno ${ }^{1,3}$, María D. Mesa ${ }^{6}$ and Concepción M. Aguilera ${ }^{6,3}$ \\ ${ }^{1}$ Growth, Exercise, NUtrition and Development (GENUD) Research Group, Universidad de Zaragoza, Health \\ Research Institute of Aragón (ISS Aragón), Food and Agriculture Institute of Aragón (IA2), Zaragoza, Spain, \\ ${ }^{2}$ Paediatric Department, Lozano Blesa University Hospital, University of Zaragoza, Food and Agriculture Institute of \\ Aragón (IA2), Zaragoza, Spain, \\ ${ }^{3}$ Spanish Biomedical Research Centre in Physiopathology of Obesity and Nutrition (CIBERobn), Instituto de Salud \\ Carlos III (ISCIII), Madrid, Spain, \\ ${ }^{4}$ Galicia Nutrition, Growth and Human Development Research Unit, Pediatric Department; Instituto de Investigación \\ Sanitaria de Santiago de Compostela (IDIS); Universidad de Santiago de Compostela, Santiago de Compostela, Spain, \\ ${ }^{5}$ Paediatric Research and Metabolism Unit, Hospital Universitario Reina Sofia; Instituto Maimónides de Investigación \\ Biomédica (IMBIC), Córdoba, Spain and \\ ${ }^{6}$ Department of Biochemistry and Molecular Biology II, Instituto de Nutrición y Tecnología de los Alimentos, \\ Universidad de Granada; Instituto de Investigación Biosanitaria ibs, Granada, Spain
}

\begin{abstract}
Introduction

Childhood obesity is associated with multiple cardio-metabolic abnormalities. A sensitive hypothesis underlying these alterations is oxidative stress, shown to be present in obesity, often accompanied by a diminished antioxidant defense. Specifically, plasma vitamin concentrations have been observed to be associated with obesity in adults and children. However, their association with cardiometabolic alterations in children is less clear.
\end{abstract}

\section{Materials and Methods}

985 children (49.2\% males, $71.7 \%$ prepubertal, $71.9 \%$ excess weight) were recruited in a case-control study of obesity in three Spanish hospitals. Pubertal status was assessed and anthropometry (weight, height), systolic and diastolic blood pressure (SBP, DBP) and serum glucose, insulin, triacylglycerols (TAG) and high-density lipoprotein cholesterol (HDL-C) were measured. Plasma concentrations of tocopherols and carotenes were determined with HPLC-MS and referred to TAG. Children were classified as MU if showing one or more of these criteria: SBP or DBP $\geq 90$ th percentile (age, sex, height), serum TAG $>90$ th percentile (age, sex), HDL-C $<10$ th percentile (age, sex), glucose $\geq 100 \mathrm{mg} / \mathrm{dL}$ or elevated HOMA-IR ( $\geq 2.5$ prepubertal, $\geq 3.38$ pubertal males, $\geq 3.905$ pubertal females). Non-fulfillment was indicative of MH status. General linear models adjusted for sex, age, recruitment center and BMI were used to evaluate differences in vitamins between $\mathrm{MH}$ and MU children.

\section{Results}

Prepubertal and pubertal children with excess weight showed lower tocopherols (Pre: $0.133 \pm 0.061$ vs $0.165 \pm 0.065$, P < 0.001; Pub: $0.120 \pm 0.057$ vs $0.163 \pm 0.066, \mathrm{P}<0.001$ ) and carotenes (Pre: $15.63 \pm 13.72$ vs $30.31 \pm 26.04, \mathrm{P}<0.001 ; \mathrm{Pub}: 12.34 \pm 9.86$ vs $22.98 \pm$ $19.25, \mathrm{P}<0.001)$ plasma concentrations than normal-weight children. MU prepubertal and pubertal children showed lower tocopherols (Pre: $0.120 \pm 0.056$ vs $0.165 \pm 0.064, \mathrm{P}<0.001$; Pub: $0.111 \pm 0.051$ vs $0.154 \pm 0.066, \mathrm{P}<0.001$ ) and carotenes (Pre: $14.07 \pm 12.61$ vs $25.97 \pm 21.94, \mathrm{P}<0.001$; Pub: $10.90 \pm 8.54$ vs $19.03 \pm 14.58, \mathrm{P}<0.001)$ plasma concentrations than MH children, independently of BMI. Individual MU components analyses showed similar associations between tocopherols and carotenes and insulin resistance, low HDL-C values and hypertriglyceridemia in prepubertal children; and between tocopherols and carotenes and elevated SBP, hyperglycemia and hypertriglyceridemia in pubertal children.

\section{Discussion}

Our findings agree with previous studies that showed decreased plasma concentrations of tocopherols and carotenes in children with obesity. However, we observe further implications of low circulating concentrations of non-enzymatic antioxidants in terms of their negative association with cardio-metabolic alterations such as insulin resistance and dyslipidemia in prepubertal and pubertal children, independently of BMI. These results must be considered when designing prevention and treatment strategies of obesity and its complications.

\section{Conflict of Interest}

There is no conflict of interest. 